\title{
sciendo
}

\section{SELECTION OF THE MOST STABLE ENDOGENOUS CONTROL GENES FOR MICRORNA QUANTITATION IN CHICKEN OVARIAN FOLLICLES $^{*}$}

\author{
Ewa Ocłoń ${ }^{1}$ Anna Hrabia ${ }^{1 *}$ \\ ${ }^{1}$ Department of Animal Physiology and Endocrinology, University of Agriculture in Krakow, \\ Al. Mickiewicza 24/28, 30-059 Kraków, Poland \\ •Corresponding author: anna.hrabia@urk.edu.pl
}

\begin{abstract}
MicroRNAs (miRNAs or miRs) belong to a class of small non-coding RNAs of 19 to 24 nucleotides long that act as negative gene regulators at the post-transcriptional level. Quantitative PCR (q-PCR) is a commonly used technique in the profiling of miRs, and identification of reliable endogenous controls is crucial for proper data normalisation. To date, no study has been performed on reference miRs for the normalisation of miR expression in chicken ovarian tissues. Therefore, the aim of the present study was to experimentally identify the most stable reference miRs for normalisation of miR q-PCR expression data in the chicken ovary. Relying on highthroughput sequencing, five putative reference miR (let-7a-3p, miR-140a-3p, miR-22-5p, miR33-5p, miR-99a-3p) were identified and subsequently analysed in a total of 66 tissue samples. The stability of candidate endogenous controls validated by the most widely used algorithms, geNorm, NormFinder, and BestKeeper, showed that let-7a-3p, miR-140a-3p, and miR-22-5p are the most appropriate choice of reference genes. Application of different normalisation approaches to the relative quantitation of randomly chosen miR-1552-5p in chicken ovarian follicles indicated the impact of the selected reference genes on miR expression. Further, the results revealed a downregulation of miR-1552-5p. In summary, the three identified endogenous reference miRs are suitable for profiling the miR expression in ovarian tissues of laying hens. Our findings provide valuable information for future miR expression studies in the avian ovary.
\end{abstract}

Key words: miRNA, reference gene, miR-qPCR, ovary, chicken

Follicle growth, development, ovulation, atresia, and regression in the chicken ovary are a well-coordinated series of events requiring reorganization of the follicular wall, which involves cell proliferation, differentiation, apoptosis, and autophagy. These processes are orchestrated by numerous gene interactions and require tight regulation of gene expression at both the transcriptional and post-transcriptional levels. A growing body of evidence indicates that microRNAs (miRNAs or miRs) play

*This work was financially supported by the National Science Centre, Poland (Grant OPUS No. UMO-2015/19/B/NZ9/01356 dedicated to A.H). 
an essential role in the post-transcriptional regulation of gene expression in various tissue types, including those of the chicken reproductive system (Lim and Song, 2014; Wang et al., 2018).

MiRs are endogenous small non-coding RNAs that act as negative regulators of gene expression (He and Hannon, 2004). Functional studies have demonstrated their critical roles in the control of a wide spectrum of biological processes such as development, oncogenesis, angiogenesis, cell cycle, differentiation, and steroidogenesis (Bartel, 2004; Mansfield et al., 2004; Burnside et al., 2008; Sirotkin et al., 2014; Nothnick, 2012; Lim and Song, 2014; Alberti and Cochella, 2017). Furthermore, various miRs have been identified in the chicken, and changes in their expression have been revealed in several processes such as embryo (Darnell et al., 2006; Glazov et al., 2008; Bannister et al., 2009; Li et al., 2012) and ovarian (Kang et al., 2013; Wang et al., 2018) development, immune organ response (Hicks et al., 2009), and disease (Tian et al., 2012; Wang et al., 2013).

Currently, several methodological approaches are available for the profiling of miRs, including next generation sequencing (NGS), chip- or bead-based arrays, and real-time quantitative polymerase chain reaction (q-PCR) (Morozova and Marra, 2008). The q-PCR method is most commonly accepted for the validation of observations determined by genome-wide profiling of miR expression, the success of which depends upon several parameters such as RNA extraction, cDNA synthesis, primer designation, amplicon detection, and data normalisation (Androvic et al., 2017). Certainly, meaningful and reproducible results, in addition to the accuracy and precision of the outcome, are mostly dependent on suitable data normalisation. Reference genes (RGs), or so-called normalisation genes, are those that are almost identically expressed in all tissues; thus differences in the expression levels of target genes that are not caused by biological variations may be estimated using these endogenous references (Meyer et al., 2010). Consequently, the use of non-stably expressed reference genes for normalisation may result in erroneous conclusions. It is generally approved that a universal reference gene suitable for all tissue types does not exist; thus, one of the most important and challenging issues in miR-qPCR experiments is the selection of stably expressed reference genes for each specific experiment, according to the tissue of interest (Schmittgen and Livak, 2008) by means of highthroughput technologies (Git et al., 2010). Despite the increasing number of studies on miR expression (Kang et al., 2013; Wang et al., 2018), there remains a lack of validation studies among avian species. Accordingly, the aim of the present investigation was to identify the stably expressed miRs in chicken ovarian tissues for use as reference genes in future miR-qPCR testing.

\section{Material and methods}

\section{Animals and tissue collection}

The animal experiment was conducted according to research protocol approved by the Local Animal Ethics Committee in Kraków, Poland (approval no. 218/2015). Hy-Line Brown laying hens were purchased from a commercial farm and caged in- 
dividually under a photoperiod of 14L:10D with free access to commercial food and water. Individual laying cycles were monitored by daily timing of oviposition, and it was found that ovulation occurred within five minutes of oviposition of the previous egg in the series.

Chickens $(n=6)$ at the age of 34 weeks were sacrificed $22 \mathrm{~h}$ prior to the predicted ovulation of the largest preovulatory follicle (F1). The ovaries were collected, placed on ice, and the following ovarian follicles were isolated: white $(>1-4 \mathrm{~mm}$ in diameter; WF); yellowish ( $>4-8 \mathrm{~mm}$; YF); small yellow ( $>8-12 \mathrm{~mm}$; SYF); three of the largest yellow preovulatory $\mathrm{F} 3-\mathrm{F} 1(\mathrm{~F} 3<\mathrm{F} 2<\mathrm{F} 1)$; white atretic; and the third postovulatory (POF3). The theca $(\mathrm{T})$ and granulosa $(\mathrm{G})$ layers were separated from the preovulatory follicles. Ovarian tissues were placed into RNAlater (Sigma-Aldrich, Saint Louis, MO, USA) for future total RNA extraction.

\section{RNA extraction and miR/smallRNA NGS}

Total RNA, including the small RNA fraction, was isolated from all collected ovarian tissues using the miRCURY RNA isolation kit (Exiqon, Qiagen, Hilden, Germany). The concentration and purity of the total RNA extracted were determined using a NanoDrop Lite (Thermo Scientifc, Madison, USA). The absorbance ratio of each sample at OD 260/280 was between 1.93 and 2.0, and the OD 260/230 was 2.03 .

The NGS service was employed to reveal a complete set of stably expressed miRs in three samples of WF (WF1, WF2, WF3), three samples of SYF (SYF1, SYF2, SYF3), three samples of granulosa layer of F2 follicle (F2G1, F2G2, F2G3), three samples of theca layer of F2 follicle (F2T1, F2T2, F2T3), and two samples of POF3 (POF3a, POF3b). The libraries were prepared according to the protocol of the NEBNext Multiplex small RNA library prep set for Illumina. Briefly, $100 \mathrm{ng}$ total RNA was converted to miR NGS libraries. During the QIAseq miRNA library construction process, each individual miR molecule was tagged with a unique molecular index (UMI). Adapters containing UMIs were ligated to the RNA, which was subsequently converted to cDNA and amplified by PCR ( 22 cycles). To allow mixing of many samples and simultaneous sequencing, the index 3'-primers were added to individual samples during the pre-amplification stage. Samples were purified following PCR. Quality control of the library preparation was performed using either a Bioanalyzer 2100 (Agilent, Santa Clara, CA, USA) or a TapeStation 4200 (Agilent). Based on the quality and concentration of the inserts, the libraries were pooled at equimolar ratios and quantitated using the KAPA library quantitation kit for Illumina (Roche, San Diego, CA, USA). MiR-seq was performed using the NextSeq500 Illumina (Illumina, San Diego, CA, USA), with an average of 10 million reads per sample and a read-length of 51 bp single-end (NGS Service Exiqon). To identify putative reference miRs in the miR/sRNA-NGS, the data obtained from three samples of each tissue type were normalised with the trimmed mean of the $M$ values (TMM) method and converted to a $\log 2$ scale. Trimming of adapters creates a distribution of sequences with different lengths. After mapping the data, each read was assigned to a class of RNA based on the length distribution. Reads representing miRs have a length of $\sim 18-23 \mathrm{nt}$, longer sequences of other origins have a length of 30-50 nt, i.e., rRNA, tRNA, mRNA, and Y-RNA fragments. Bioinformatics analy- 
sis was performed by searching against the miRBase, version 20.0 to identify known mature miRs. In NGS experiments, the expression level of miRs is measured as tags per million (TPM). The number of reads for a particular miR is divided by the total number of mapped reads and multiplied by one million.

\section{miR expression analysis}

For miR detection, $10 \mathrm{ng}$ RNA from each tissue sample was reverse transcribed (RT) in technical duplicates using a TaqMan microRNA reverse transcription kit (Thermo Fisher Scientific, Waltham, MA, USA) with TaqMan microRNA primers specific for the miRNAs of interest, following the manufacturer's instructions. Briefly, $10 \mathrm{ng} / \mu \mathrm{L}$ RNA, $1 \mathrm{X}$ stem-loop RT primer, $3.33 \mathrm{U} / \mu \mathrm{L}$ reverse transcriptase, $0.25 \mathrm{U} / \mu \mathrm{L}$ RNase inhibitor, $0.25 \mathrm{mM}$ dNTPs, and $1 \mathrm{X}$ reaction buffer were combined in a total reaction volume of $15 \mu \mathrm{L}$ and incubated at $16^{\circ} \mathrm{C}$ for $30 \mathrm{~min}, 42^{\circ} \mathrm{C}$ for $30 \mathrm{~min}$, and $85^{\circ} \mathrm{C}$ for $5 \mathrm{~min}$.

Following the RT step, $1.33 \mu \mathrm{L}$ RT reaction was combined with $1 \mu \mathrm{L}$ TaqMan MicroRNA Assay (20X), $10 \mu \mathrm{L}$ TaqMan ${ }^{\circledR}$ Universal PCR Master Mix, No AmpErase $^{\circledR} \mathrm{UNG}$, and $7.67 \mu \mathrm{L}$ nuclease free water in a final volume of $20 \mu \mathrm{L}$. Real-time PCR was performed using an Applied Biosystems StepOnePlus real-time PCR system with cycling conditions of $95^{\circ} \mathrm{C}$ for $10 \mathrm{~min}$ followed by 40 cycles of $95^{\circ} \mathrm{C}$ for $15 \mathrm{~s}$ and $60^{\circ} \mathrm{C}$ for $60 \mathrm{~s}$.

The impact of normalisation strategy on target miRNA profiles in collected ovarian tissues ( $\mathrm{n}=11$ tissues per each ovary) was evaluated in the exemplifying case of miR-1552-5p. The expression levels of miR-1552-5p were computed using the best pairs of genes sorted by the geNorm, NormFinder, and BestKeeper applets. The normalised relative quantity of miR-1552-5p was calculated using the Pfaffl method (Pfaffl, 2001).

\section{Calculations of the stability of endogenous controls and statistical tests}

The stability of reference genes was assessed using the three most common algorithms, geNorm (Vandesompele et al., 2002), NormFinder (Andersen et al., 2004), and BestKeeper (Pfaffl et al., 2004).

The geNorm applet estimates the stability of candidate reference genes based on the average pairwise variation of a gene compared with that of all other genes. Subsequently, it determines the optimal number of reference genes required by analyzing the pairwise variation $\left(\mathrm{V}_{\mathrm{n} / \mathrm{n}+1}\right)$ among candidate genes. The gene stability value (M) and the pairwise variation value (V) were calculated by the geNorm software using $2^{-\Delta \mathrm{CqMin}}$ values. The $\Delta \mathrm{Cq}_{\mathrm{Min}}$ value was calculated using the following equation: $\Delta \mathrm{Cq}_{\mathrm{Min}}=$ Target gene $\mathrm{Cq}-\mathrm{Min} \mathrm{Cq}$, where Min Cq is the lowest $\mathrm{Cq}$ value of a candidate gene. According to the stability measure, an $\mathrm{M}$ value of 1.5 was defined as the upper limit for a candidate reference gene and 0.5 was the $\mathrm{M}$ value typically noticed for stably expressed reference genes in homogeneous sample groups, whereas values between 0.5 and 1 were observed when evaluating endogenous controls in heterogeneous samples (Bustin et al., 2009).

NormFinder analysis is based on an ANOVA model that considers intra- and inter-group variabilities, ranking the set of candidate normalisation genes according 
to their expression stability. In NormFinder, the $\mathrm{Cq}$ values are converted to a linear scale according to the formula $\mathrm{E}^{-\Delta \mathrm{Cq}}$, which are log-transformed by the program itself. The lowest stability value indicates the most stably expressed gene.

BestKeeper analysis determines the most stably expressed gene based on the Pearson correlation coefficient (r) of the BestKeeper index (BI), which is the geometric mean (GM) of the Cq values of candidate genes. Based on the raw $\mathrm{Cq}$ value of each sample, the arithmetic mean (AM), minimal (Min) and maximal (Max) values, standard deviation (SD), and coefficient of variance (CV) are also calculated.

A paired Student t-test was used to compare the expression values of miR-1552-5p between samples derived from the same tissue but normalised with different pairs of reference genes. Statistical analysis was performed with GraphPad Prism 4.0 (GraphPad Software, San Diego, CA, USA). P-values of $<0.05$ were considered statistically significant.

\section{Results}

High-throughput sequencing yielded an approximate 11,395,437 raw reads for each sample. After filtering out low quality sequences, on average 6.6 million reads were obtained per sample and the average genome mapping rate was $90.7 \%$ (Table 1). According to entries in miRBase_20, 128 miRNAs were detected ( $\geq 10$ TPM) in all samples (data not shown). The length distribution for each class of RNAs was similar in all samples (Figure 1), and the majority of them changed from 18 to $23 \mathrm{nt}$, which was consistent with the typical size range of miRNAs.

Table 1. Summary of the mapping results for each sample

\begin{tabular}{l|c|c|c|c|c|c}
\hline \multicolumn{1}{c}{ Sample } & Raw reads & Clean reads & $\begin{array}{c}\text { miRNA/ small } \\
\text { RNA }\end{array}$ & Genome & Outmapped & Unmapped \\
\hline WF 1 & $11,327,858$ & $6,902,914$ & $65.3 \%$ & $22.5 \%$ & $3 \%$ & $8.9 \%$ \\
WF 2 & $11,622,222$ & $6,583,453$ & $74.3 \%$ & $10.9 \%$ & $2.7 \%$ & $11.8 \%$ \\
WF 3 & $10,916,446$ & $7,204,677$ & $60.8 \%$ & $20.6 \%$ & $8.3 \%$ & $10.1 \%$ \\
SYF 1 & $13,025,563$ & $8,146,107$ & $65.3 \%$ & $21.8 \%$ & $7.4 \%$ & $5.3 \%$ \\
SYF 2 & $11,427,209$ & $4,773,341$ & $49.1 \%$ & $27.8 \%$ & $13.5 \%$ & $9.3 \%$ \\
SYF 3 & $11,004,397$ & $5,094,499$ & $51.2 \%$ & $30.6 \%$ & $12.6 \%$ & $5.4 \%$ \\
F2T 1 & $12,548,112$ & $8,259,958$ & $70.6 \%$ & $21.8 \%$ & $2.0 \%$ & $5.3 \%$ \\
F2T 2 & $10,894,005$ & $6,633,825$ & $49.3 \%$ & $27.2 \%$ & $15.4 \%$ & $7.9 \%$ \\
F2T 3 & $10,230,869$ & $5,467,596$ & $75.4 \%$ & $9.2 \%$ & $2.3 \%$ & $12.9 \%$ \\
F2G 1 & $10,220,699$ & $4,811,606$ & $55.8 \%$ & $25.5 \%$ & $7.6 \%$ & $10.9 \%$ \\
F2G 2 & $10,051,575$ & $5,291,118$ & $72.3 \%$ & $9.7 \%$ & $2.3 \%$ & $15.4 \%$ \\
F2G 3 & $12,187,812$ & $7,164,704$ & $59.7 \%$ & $22.4 \%$ & $8.2 \%$ & $9.5 \%$ \\
POF3a & $13,686,777$ & $9,068,378$ & $60.3 \%$ & $24.0 \%$ & $9.6 \%$ & $5.8 \%$ \\
POF3b & $10,392,585$ & $6,634,613$ & $65.3 \%$ & $21.5 \%$ & $5.4 \%$ & $7.6 \%$ \\
\hline
\end{tabular}

${ }^{1} \mathrm{WF}$ - white follicles, ${ }^{2} \mathrm{SYF}$ - small yellow follicles, ${ }^{3} \mathrm{~F} 2$ - the second largest yellow preovulatory follicle, ${ }^{4} \mathrm{G}$ - granulosa layer, ${ }^{5} \mathrm{~T}$ - theca layer, ${ }^{6} \mathrm{POF} 3$ - the third postovulatory follicle. 


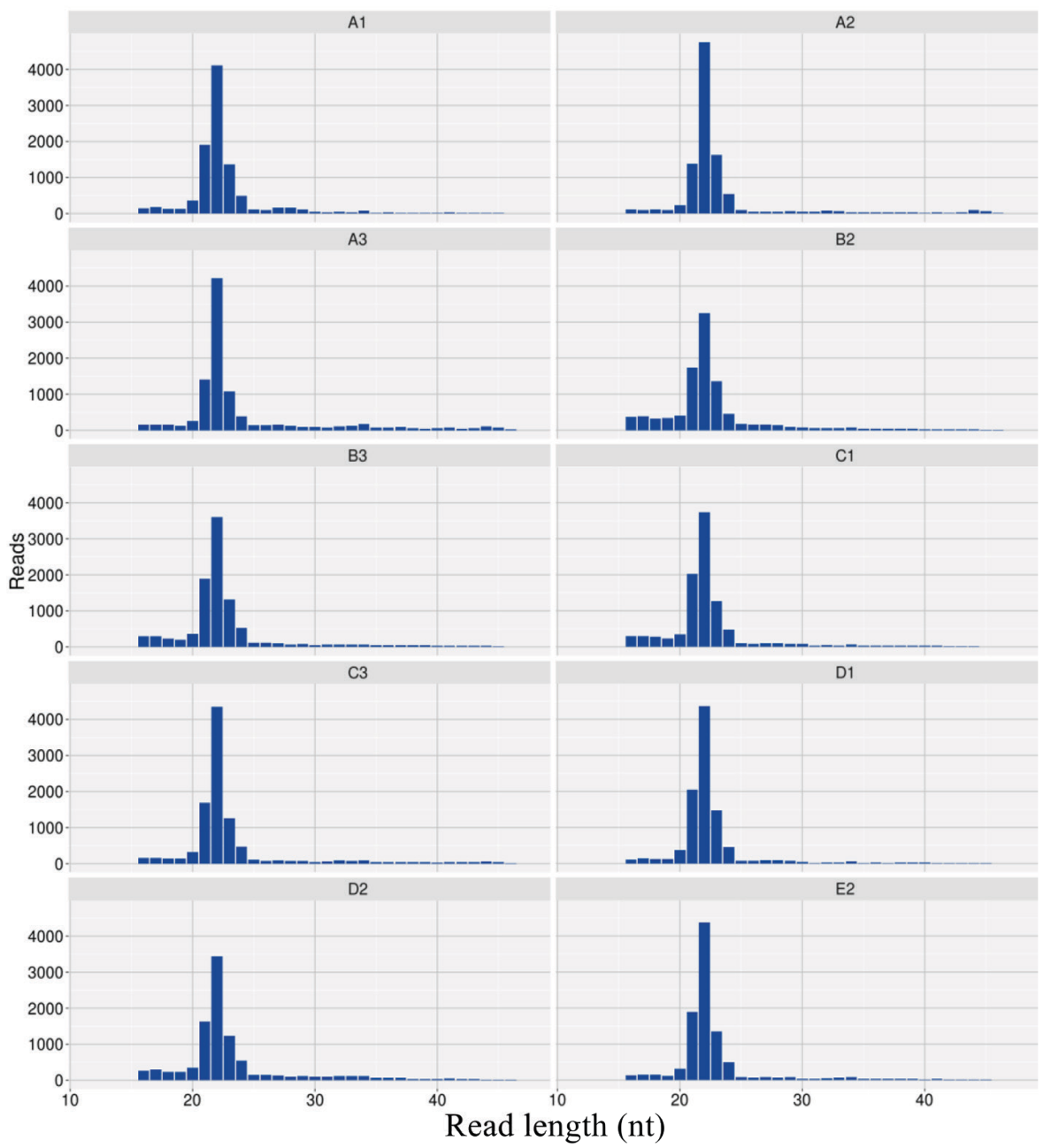

MicroRNA appeared as a peak around 18-23 nt in tissue samples of the white follicles (A1, A2, A3), small yellow follicles (B2, B3), theca layer of F2 follicle (C1, C3), granulosa layer of F2 follicle (D1, D2), and third postovulatory follicle (E2).

Figure 1. The exemplified histograms of RNA-seq read length distributions

Based on the NormFinder algorithm, NGS highlighted the 25 most stably expressed miRs across the sample set, which therefore may be good candidates for normalisation. We chose randomly five (Table 2) for further q-PCR validation. According to the quantifiability criterion for q-PCR analysis, the raw Cq values were determined, and manual thresholds were applied to each miR. The gene to gene differences among the $\mathrm{Cq}$ values $(\mathrm{Cq}<35)$ were relatively similar for all the tested follicles. 
Table 2. Characteristics of the five candidate reference miRs in chicken ovarian follicles

\begin{tabular}{c|l|c|c|c|c|c}
\hline miR symbol & \multicolumn{1}{c|}{ Sequence } & Accession number & $\begin{array}{c}\text { Average } \\
\text { TPM }\end{array}$ & $\begin{array}{c}\text { Average } \\
\text { stability }\end{array}$ & $\begin{array}{c}\text { Stability/ } \\
\text { TPM }\end{array}$ & $\begin{array}{c}\text { PCR } \\
\text { efficiency }\end{array}$ \\
\hline gga-let-7a-3p & $\begin{array}{l}\text { CTATACAATC- } \\
\text { TACTGTCTTTCC }\end{array}$ & MIMAT0026491 & 60 & 2.20 & 0.04 & 1.91 \\
gga-miR-22-5p & $\begin{array}{l}\text { AGTTCTTCAGTG- } \\
\text { GCAAGCTTTA }\end{array}$ & MIMAT0007287 & 142 & 7.93 & 0.06 & 1.92 \\
gga-miR-140a-3p & $\begin{array}{l}\text { CCACAGGGTA- } \\
\text { GAACCACGGAC }\end{array}$ & MIMAT0003722 & 133 & 11.82 & 0.08 & 1.86 \\
gga-miR-33-5p & $\begin{array}{l}\text { GTGCATTGTAGTT- MIMAT0001100 } \\
\text { GCATTGC }\end{array}$ & 48 & 4.36 & 0.09 & 1.84 \\
gga-miR-99a-3p & $\begin{array}{l}\text { CAAGCTCGCTTC- MIMAT0006781 } \\
\text { TATGGGTCT }\end{array}$ & 120 & 11.24 & 0.09 & 1.89 \\
\hline
\end{tabular}

${ }^{1} \mathrm{TPM}$ - in NGS experiments, the expression level of miRs is measured as tags per million.

\section{Expression stability of putative endogenous control genes}

The GeNorm and NormFinder applets were used to compare and grade the five potential reference miRs based on their stability in chicken ovarian follicles. geNorm calculates the gene stability $M$ value and ranks candidate reference miRs from the least stable (highest $M$ value) to the most stable (lowest $M$ value). As shown in Figure 2, miR-22-5p and miR-140a-3p $(\mathrm{M}=0.58)$ were identified as the most stable miRs, followed by let-7a-3p $(\mathrm{M}=0.69)$, miR-99a-3p $(\mathrm{M}=0.82)$, and miR-33-5p $(\mathrm{M}=0.93)$. Furthermore, the geNorm applet also calculates the optimal number of miRs needed for reliable normalisation of q-PCR data, based on the variable $\mathrm{V}$ value as the pairwise variation between two sequential normalisation factors $\left(\mathrm{NF}_{n}\right.$ and $\left.\mathrm{NF}_{\mathrm{n}+1}\right)$, where the lowest $\mathrm{V}_{\mathrm{n} / \mathrm{n}+1}$ indicates the optimal number of references genes (Figure 3). The cut-off value for the lowest $\mathrm{V}_{\mathrm{n} / \mathrm{n}+1}$ is 0.15 , below which the additional reference gene is not required for normalisation (Vandesompele et al., 2002); however, it must be noted that 0.15 is not a strict cut-off value, and the optimal number of reference genes can be estimated according to the requirements of the investigation. In the present study, the highest decrease in variation was achieved with the fourth most stable miR $\left(\mathrm{V}_{4 / 5}=0.197\right.$; Figure 3$)$, indicating that a suitable normalisation strategy for relative gene quantitation should include at least four endogenous references.

NormFinder ranked the miRs as follows: miR-140a-3p $(M=0.213)$, let-7a-3p $(\mathrm{M}=0.260)$, miR-22-5p $(\mathrm{M}=0.285)$, miR-99a-3p $(\mathrm{M}=0.297)$, and miR-33-5p $(\mathrm{M}=0.368)$ (Figure 4). miR-140a-3p and let-7a-3p were recommended as the best combination $(\mathrm{M}=0.163)$, and miR-140a-3p was recommended as the single best normalizer. Additionally, combination of miR-140a-3p with either miR-22-5p or miR-99a-3p led to stability $M=0.176$ or $M=0.177$, while combination let-7a-3p with either miR-99a-3p or miR-22-5p led to a stability $\mathrm{M}=0.183$ and $\mathrm{M}=0.199$, respectively. Notwithstanding, the intra- and inter-group variations independently calculated for each tissue with NormFinder are shown in Figure 5. The bars in the graph illustrate the inter-group variation, and the error bars illustrate the average of intra-group variation. The highest ranking candidate gene is the one with a minimal 
inter-group variation, and at the same time, the smallest possible error. Thus, the best overall performance was shown by miR-140a-3p (YF, SYF, F3T, F3G, F2G, F1G), which displayed low values of both inter-group (an average 0.013) and intra-group variability (an average 0.027). Overall performance was low also for miR-22-5p (WF, F1T) and let-7a-3p (POF3, ATR), which displayed low inter-group variation (an average $0.010 ;-0.022$ ) and the intermediate intra-group variability (an average $0.054 ; 0.050)$.

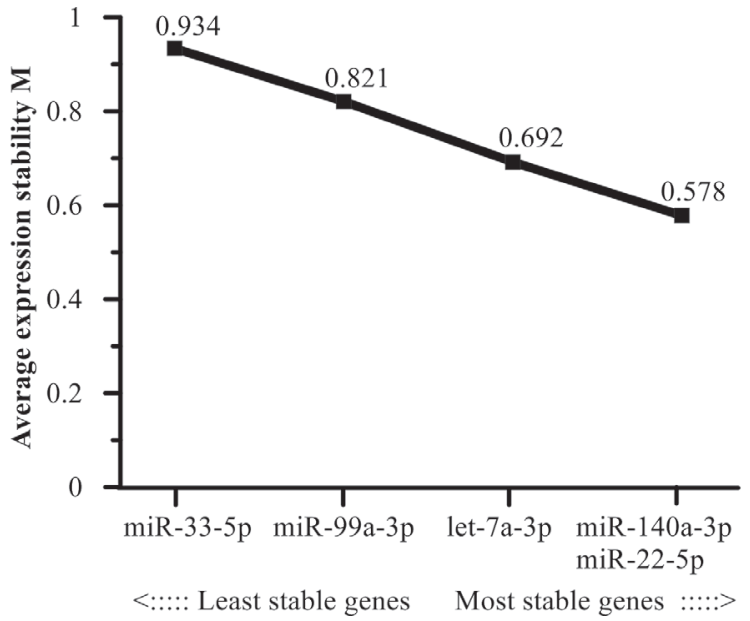

Figure 2. Average expression stability values of reference genes evaluated by geNorm $(n=66$ tissue samples)

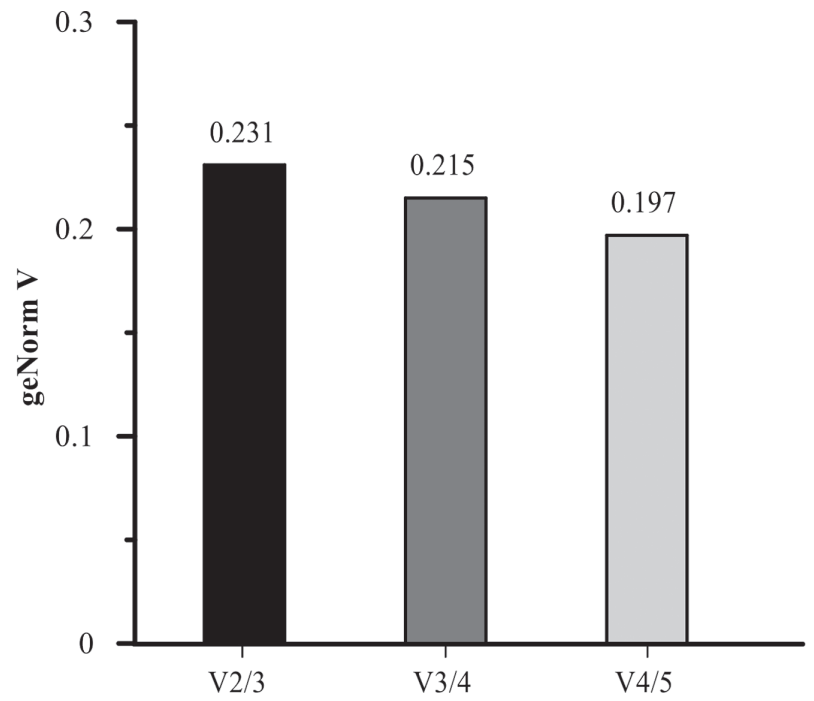

Figure 3. Pairwise variation $\left(\mathrm{V}_{\mathrm{n}} / \mathrm{V}_{\mathrm{n}+1}\right)$ generated by geNorm for the determination of the optimal number of reference genes $(\mathrm{n}=66$ tissue samples $)$ 


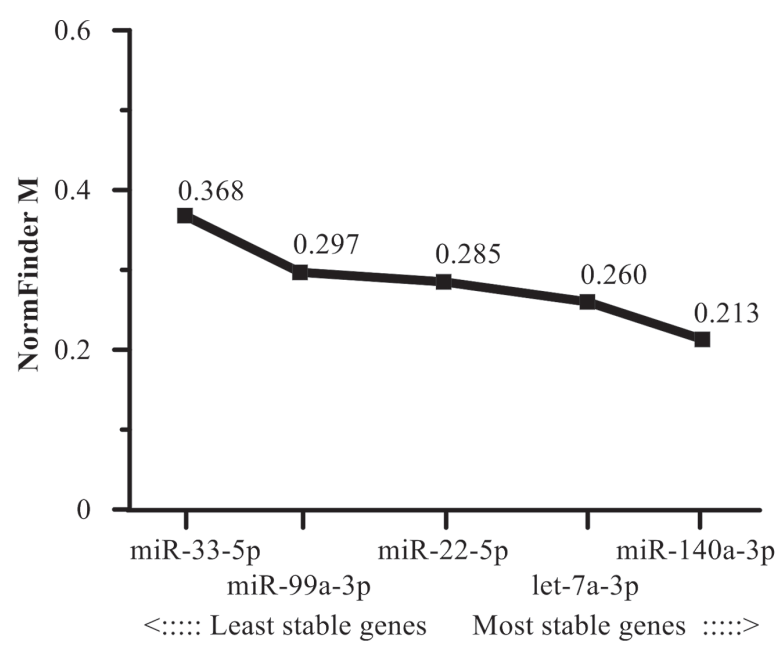

Figure 4. Reference gene stability values estimated by NormFinder $(n=66$ tissue samples)

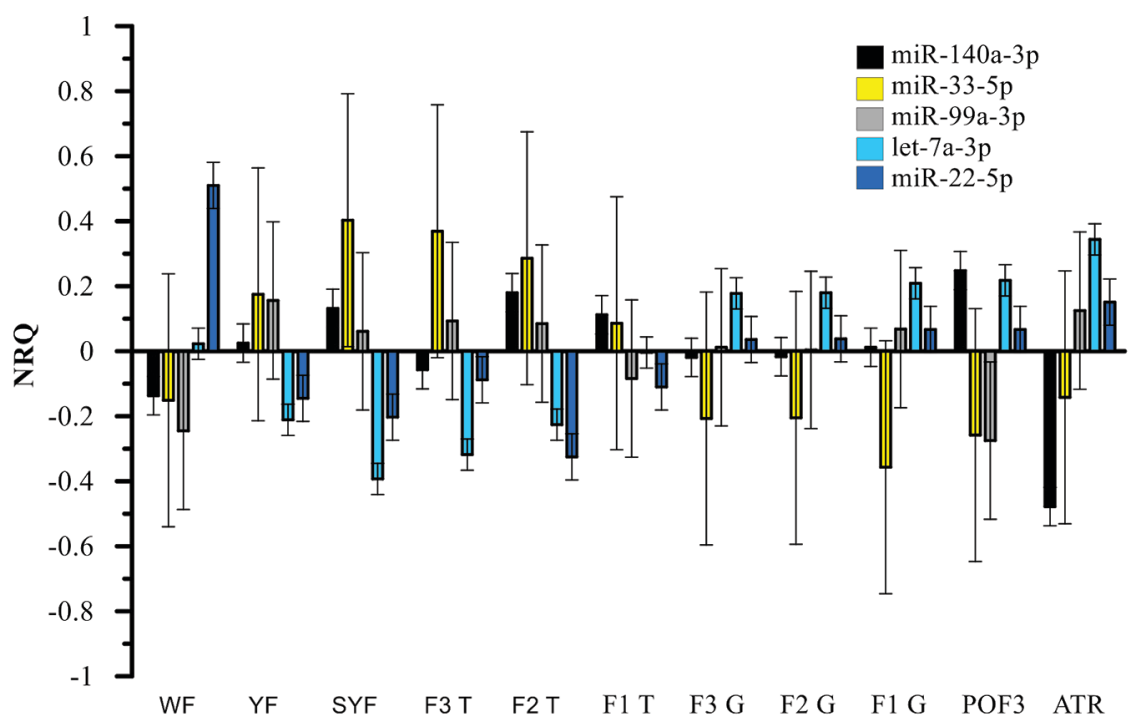

Abbreviations: NRQ - Normalised Relative Quantity, WF - white follicles, YF - yellowish follicles, $\mathrm{SYF}$ - small yellow follicles, F3-F1 $(\mathrm{F} 3<\mathrm{F} 2<\mathrm{F} 1)$ - the 3 largest yellow preovulatory follicles, $\mathrm{G}$ - granulosa layer, T - theca layer, POF3 - the third postovulatory follicle, ATR - atretic follicles.

Figure 5. Inter- and intra-group variation evaluated by NormFinder. The bars illustrate the inter-group variation and the error bars illustrate the average intra-group variation 
Table 3. Descriptive statistics of the five candidate reference genes based on their crossing point (CP) values

\begin{tabular}{l|c|c|c|c|c}
\hline \multicolumn{1}{c}{ Factor } & miR-140a-3p & miR-33-5p & miR-99a-3p & miR-22-5p & let-7a-3p \\
\hline GM (CP) & 34.45 & 28.43 & 33.19 & 28.03 & 28.69 \\
AM (CP) & 34.46 & 28.46 & 33.21 & 28.05 & 28.70 \\
Min (CP) & 32.67 & 24.72 & 31.08 & 26.14 & 26.39 \\
Max (CP) & 36.00 & 30.92 & 35.59 & 29.97 & 29.99 \\
SD ( \pm CP) & 0.69 & 0.97 & 0.97 & 0.90 & 0.83 \\
CV (\% CP) & 2.00 & 3.41 & 2.91 & 3.21 & 2.88 \\
\hline
\end{tabular}

${ }^{1} \mathrm{GM}(\mathrm{CP})$ - the geometric mean of $\mathrm{CP},{ }^{2} \mathrm{AM}(\mathrm{CP})$ - the arithmetic mean of $\mathrm{CP},{ }^{3} \mathrm{Min}(\mathrm{CP})$ and $\mathrm{Max}(\mathrm{CP})-$ the extreme values of $\mathrm{CP},{ }^{4} \mathrm{SD}( \pm \mathrm{CP})$ - the standard deviation of the $\mathrm{CP},{ }^{5} \mathrm{CV}(\% \mathrm{CP})$ - the coefficient of variance expressed as a percentage on the CP level.

Table 4. Correlation analysis of reference genes (RGs) versus the BestKeeper (BK) index

\begin{tabular}{l|c|c|c|c|c}
\hline & $\begin{array}{c}\text { miR-140a-3p } \\
\text { vs. BK }\end{array}$ & $\begin{array}{c}\text { miR-33-5p } \\
\text { vs. BK }\end{array}$ & $\begin{array}{c}\text { miR-99a-3p } \\
\text { vs. BK }\end{array}$ & $\begin{array}{c}\text { miR-22-5p } \\
\text { vs. BK }\end{array}$ & $\begin{array}{c}\text { let-7a-3p } \\
\text { vs. BK }\end{array}$ \\
\hline Coeff. of corr. (r) & 0.460 & 0.462 & 0.548 & 0.786 & 0.543 \\
Coeff. of det. ( $\left.\mathrm{r}^{\wedge} 2\right)$ & 0.240 & 0.213 & 0.300 & 0.487 & 0.295 \\
P-value & 0.001 & 0.001 & 0.001 & 0.001 & 0.001 \\
Power (x-fold) & 1.62 & 2.06 & 2.11 & 2.41 & 1.82 \\
\hline
\end{tabular}

Further, the gene expression variation was calculated for all five candidate reference genes based on $\mathrm{Cq}$ values and is displayed as the standard deviation (SD) and coefficient of variance $(\mathrm{CV})$ by BestKeeper. The lowest SD values indicate the highest stability $(<1)$. BestKeeper highlighted miR-140a-3p as the reference gene with the lowest variation among all candidates, with an SD of 0.69 and a CV value of 2.0 throughout all examined ovarian tissues (Table 3 ). The grading of the candidate reference genes from the most stable (lowest SD) to the least stable (highest SD) was miR-140a-3p $>$ let-7a-3p $>$ miR-22-5p $>$ miR-33-5p and miR-99a-3p. However, the best stability value of individual reference genes, calculated as the best pairwise correlation between genes and BI was obtained for miR-22-5p $(r=0.786)$, with a P value of 0.001 (Table 4).

\section{Effect of endogenous control genes on the measure of relative mir-1552-5p expression level}

To verify the results of the reference gene selection analysed by geNorm, NormFinder, and Bestkeeper, the expression of miR-1552-5p, chosen randomly, was calculated using each gene independently (Figure 6), and the best pairs of genes were sorted by geNorm and NormFinder (Figure 7). Normalisation to the most stable pair (miR-140a-3p/miR-22-5p or miR-140a-3p/let-7a-3p) revealed either lower or unchanged expression of miR-1552-5p in all ovarian follicles, respectively, with the exception of the granulosa layer of the second largest yellow preovulatory follicle (F2) (Figure 7). In contrast, when miR-1552-5p was normalised to the least stable 
gene, either miR-99a-3p or miR-33-5p, expression in the WF, theca layer of F3, and the granulosa layer of F3-F1 was interpreted as either higher or unchanged (Figure 6).

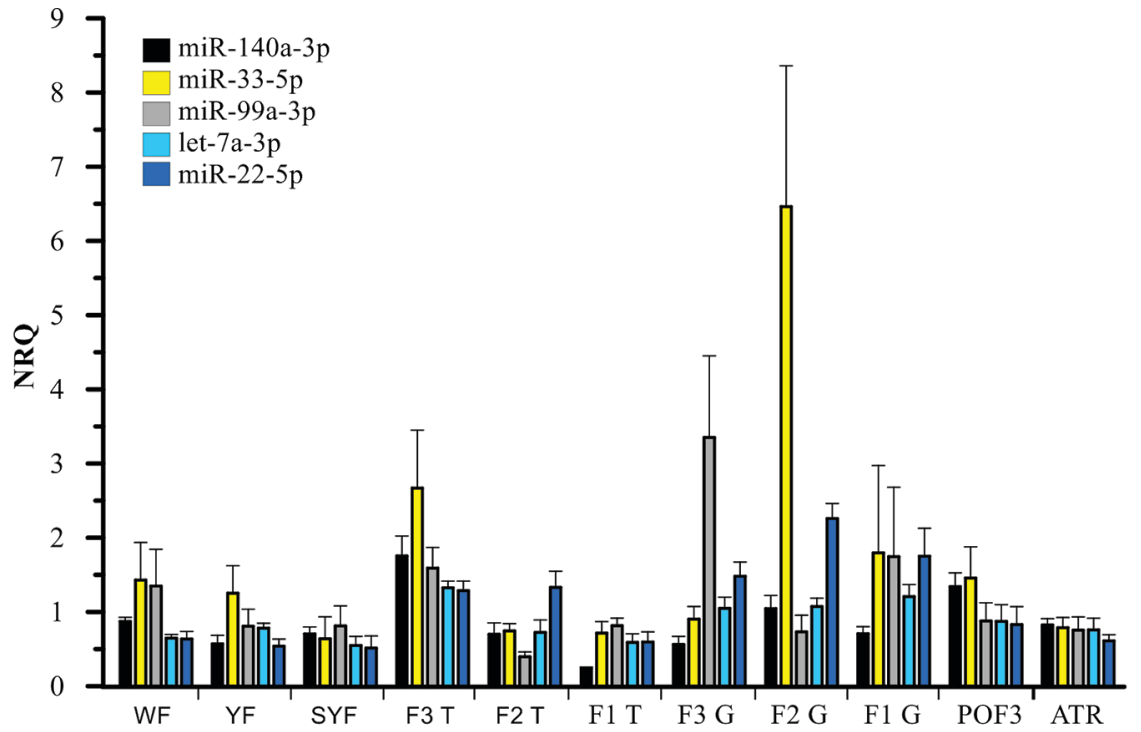

Figure 6. Relative quantitation of miR-1552-5p gene expression using reference genes for normalisation in the chicken ovary. Each value represents the mean \pm SEM from six determinations. Abbreviations as in Figure 5

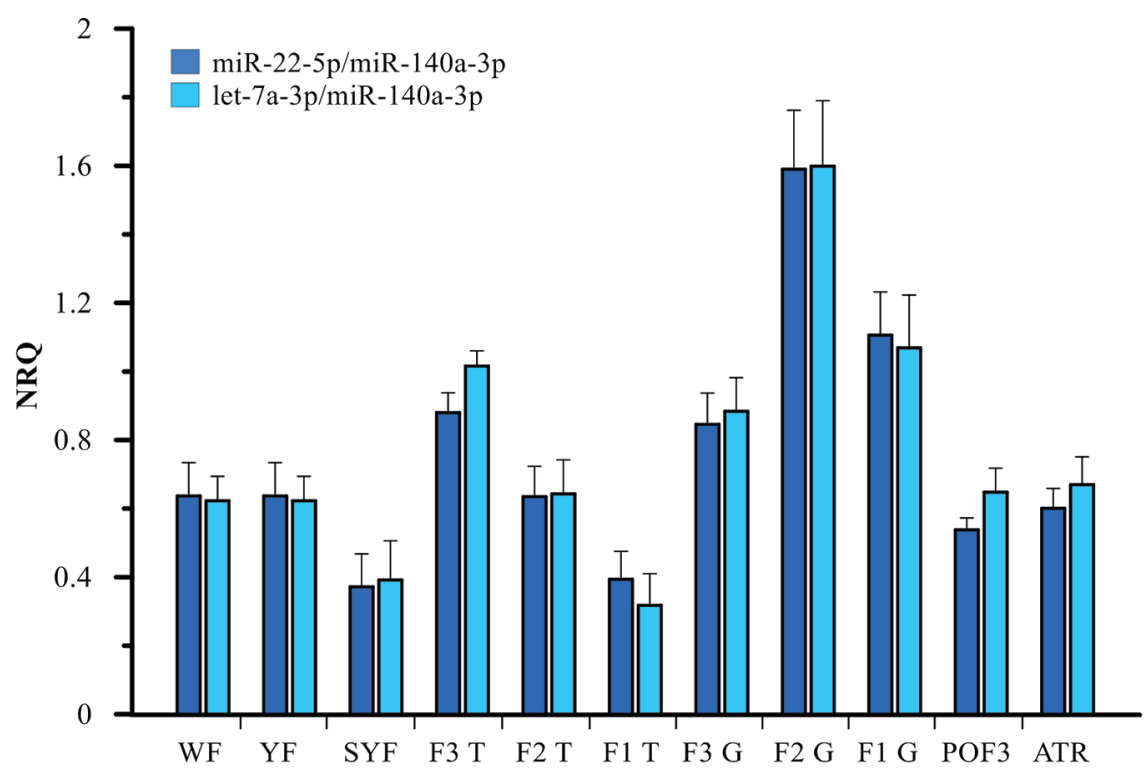

Figure 7. Normalised relative quantitation of miR-1552-5p gene expression using the best pair combination of reference genes generated by geNorm and NormFinder. Abbreviations as in Figure 5 


\section{Discussion}

The genome sequence obtainable for chicken is readily available, which improves its use as a model for the investigation of functional genomics. An increasing number of studies have demonstrated that miRs serve as important regulators of numerous biological processes in chickens (Lim and Song, 2014; Wu et al., 2017; Liu et al., 2018; Fang et al., 2018); however, it should be highlighted that performing miR expression analysis requires correction of technical variations among experiments. This correction is most extensively achieved by the use of endogenous control genes, which are stably expressed across samples, as reference genes for normal expression in a specific tissue. Thus, prior to examination of q-PCR-based miR expression, normalisation should be implemented to minimize technical-associated effect and remove systematic bias and experimental variation. It is widely accepted that a single universal reference gene does not exist, and thus, searching for stably expressed genes in each experimental system is recommended. To the best of our knowledge, no widely accepted reference genes have been reported for the normalisation of miR q-PCR data in the chicken ovary. Therefore, in the present study we identify the most stable reference miRs for normalisation of miR q-PCR expression data in the ovary of laying hens. The starting point was miR-NGS analysis, currently the most accurate technology for miR profiling. Thus, the candidate reference genes were identified based solely on the empirical RNA-Seq data. NGS analysis revealed 25 miRs, that were most stably expressed across the tissues, and thus, were good as normalizers in q-PCR validation. Of the 25 miRs suggested, five miRs (miR-33-5p, miR-99a-3p, miR-140a-3p, miR-22-5p, and let-7a-3p) were subsequently examined.

The most stable reference genes generated from the most commonly used applets, NormFinder, geNorm, and BestKeeper (Vandesompele et al., 2002; Meyer et al., 2010) showed a high level of similarity; however, there were slight differences in the rank order. For instance, across all ovarian tissues, the top two stably expressed genes were miR-140-3p and miR-22-5p for geNorm but miR-140a-3p and let-7a-3p for NormFinder. According to BestKeeper, the two most stable miRs were miR$140 a-3 p$ and let-7a-3p based on the SD values. On the other hand, considering correlation coefficient (r), miR-22-5p and let-7a-3p were the most suitable genes with constant expression. This deviation is natural due to the different algorithms used. Based on the obtained data, we propose miR-140a-3p, miR-22-5p, and let-7a-3p as normalizers for further studies.

The practical consequences of miR normalisation were subsequently evaluated using gga-miR-1552-5p as a case study, given the evidence that miR-1552-5p is mostly downregulated in chicken ovarian tissues, with the exception of the granulosa layer of the largest yellow preovulatory F2 follicle. Recently, significant changes in miR-1552-5p expression (downregulation) were confirmed in skin tissue of earlyand late-feathering birds (Fang et al., 2018).

Profiling of miR has been applied to avian reproductive system tissues with a view to identifying miRs associated with atrophy of the chicken ovary (Liu et al., 2018) or high rates of egg production (Wu et al., 2017) or identifying novel and known miRs that are differentially expressed in the ovaries of laying and broody 
geese (Xu et al., 2014). In all these studies, as well as in systematic research on the regulatory functions of miRs in chicken hepatic lipid metabolism (Li et al., 2016) or skin follicle development in early-feathering and late-feathering chickens (Fang et al., 2018), reference genes were selected based on available literature or suggestions by manufacturers. In above mentioned studies the most commonly used reference gene was U6 snRNA. Eventually, we realised that the struggle, cost, and sample requirements necessary for the experimental selection of miR normalizers is not always achievable. Under these circumstances, the data presented herein strongly suggest that a combination of miR-140a-3p, miR-22-5p, and let-7a-3p is a reasonable choice of reference genes for the investigation of miRs in the ovary of laying hens. Undoubtedly, however, an empirical validation is the optimal strategy to ensure proper miR quantitation by q-PCR.

In conclusion, based on miR-NGS analysis and q-PCR validation, we identified three suitable endogenous reference miRs that can be used to evaluate miR expression in chicken ovarian follicles. Our findings provide valuable information for future miR expression studies in the avian ovary.

\section{Acknowledgements}

The authors are grateful to Dorota Katarzyńska-Banasik from the University of Agriculture in Krakow for statistical consulting and Maria Mika, Joanna K. Socha, and Kinga Kowalik for valuable help during experiment.

\section{Disclosure statement}

No potential conflict of interest was reported by the authors.

\section{Author contributions}

$\mathrm{EO}$ and $\mathrm{AH}$ devised the project and the main conceptual ideas. EO performed analyses, while AH designed figures. AH received funding. Both authors analysed data, discussed the results and contributed to the final manuscript.

\section{References}

A l berti C., Cochella L. (2017). A framework for understanding the roles of miRNAs in animal development. Development, 144: 2548-2559.

Anders e n C.L., J e n s e n J.L., Ø rn t o ft T.F. (2004). Normalisation of real-time quantitative reverse transcription-PCR data: a model-based variance estimation approach to identify genes suited for normalisation, applied to bladder and colon cancer data sets. Cancer Res., 64: 5245-5250.

Androvic P., Valihrach L., Elling J., Sjoback R., Kubista M. (2017). Two-tailed RTqPCR: a novel method for highly accurate miRNA quantification. Nucleic Acids Res., 45:e144.

B a nn ister S.C., Tizard M.L., D or an T.J., S in c la ir A.H., S m i th C.A. (2009). Sexually dimorphic microRNA expression during chicken embryonic gonadal development. Biol. Reprod., 81: $165-176$.

B a r t e 1 D.P. (2004). MicroRNAs: genomics, biogenesis, mechanism, and function. Cell, 116: 281-297. Burnside J., Ouyang M., Anderson A., Bernberg E., Lu C., Meyers B.C., Gree n P.J., Mark is M., I s a c s G., H u a ng E., M organ R.W. (2008). Deep sequencing of chicken microRNAs. BMC Genomics, 9: 185. 
Bustin S.A., B en es V., Gars on J.A., Hellemans J., Huggett J., Kubista M., Mueller R., Nolan T., Pfaffl M.W., Shipley G.L., Vandesompele J., Wittwer C.T. (2009). The MIQE guidelines: minimum information for publication of quantitative real-time PCR experiments. Clin. Chem., 55: 611-622.

D a rnell D.K., Kaur S., S t a n is law S., Konie c zk a J.H., Yat s k i evy ch T.A., A n tin P.B. (2006). MicroRNA expression during chick embryo development. Dev. Dynam., 235: 3156-165.

F a n g G., Ji a X., Li H., Ta n S., N i e Q., Yu H., Yang Y. (2018). Characterization of microRNA and $\mathrm{mRNA}$ expression profiles in skin tissue between early-feathering and late-feathering chickens. BMC Genomics, 19: 399.

Git A., Dvinge H., S a lmon-Divon M., Osborne M., Kutter C., Hadfield J., Bert on e P., Cald a s C. (2010). Systematic comparison of microarray profiling, real-time PCR, and next-generation sequencing technologies for measuring differential microRNA expression. RNA, 16: $991-1006$.

Glazov E.A., Cottee P.A., B arris W.C., Moore R.J., Dalrymple B.P., Tizard M.L. (2008). A microRNA catalog of the developing chicken embryo identified by a deep sequencing approach. Genome Res., 18: 957-964.

H e L., Han n on G.J. (2004). MicroRNAs: small RNAs with a big role in gene regulation. Nat. Rev. Genet., 5: 522-531.

Hicks J.A., Te mbhurne P.A., Li u H.C. (2009). Identification of microRNA in the developing chick immune organs. Immunogenetics, 61: 231-240.

Kang L., Cui X., Zhang Y., Yang C., Jiang Y. (2013). Identification of miRNAs associated with sexual maturity in chicken ovary by Illumina small RNA deep sequencing. BMC Genomics, 14:e352.

Li H., Ma Z., Ji a L., Li Y., Xu C., Wang T., Han R., Jiang R., Li Z., Sun G., Kang X., L i u X. (2016). Systematic analysis of the regulatory functions of microRNAs in chicken hepatic lipid metabolism. Sci. Rep., 6: 31766.

Li T., Wang S., Wu R., Zh ou X., Z hu D., Zhang Y. (2012). Identification of long non-protein coding RNAs in chicken skeletal muscle using next generation sequencing. Genomics, 99: 292-298.

L i m W., S o n g G. (2014). Identification of novel regulatory genes in development of the avian reproductive tracts. PLosOne, 9(4):e96175.

Liu L., Xiao Q., Gilbert E.R., Cui Z., Zhao X., Wang Y., Yin H., Li D., Zhang H., $\mathrm{Zh} \mathrm{u} \mathrm{Q.} \mathrm{(2018).} \mathrm{Whole-transcriptome} \mathrm{analysis} \mathrm{of} \mathrm{atrophic} \mathrm{ovaries} \mathrm{in} \mathrm{broody} \mathrm{chickens} \mathrm{reveals} \mathrm{regu-}$ latory pathways associated with proliferation and apoptosis. Sci. Rep., 8: 7231.

Mansfield J.H., Harfe B.D., Nissen R., Obenauer J., Srineel J., Chaudhuri A., Farzan-Kashani R., Zuker M., Pasquinelli A.E., Ruvkun G., Sharp P.A., Tab in C.J., M c Manus M.T. (2004). MicroRNA responsive 'sensor' transgenes uncover Hox-like and other developmentally regulated patterns of vertebrate microRNA expression. Nat. Genet., 36: 1079-1083.

M e y e r S.U., Pfaffl M.W., Ulbrich S.E. (2010). Normalisation strategies for microRNA profiling experiments: a 'normal' way to hidden layer of complexity? Biotechnol. Lett., 33: 1777-1788.

Morozova O., Marra M.A. (2008). Applications of next-generation sequencing technologies in functional genomics. Genomics, 92: 255-264.

N o th n i c k W.B. (2012). The role of micro-RNAs in the female reproductive tract. Reproduction, 143: 559-576.

P faffl M.W. (2001). A new mathematical model for relative quantification in real-time RT-PCR. Nucleic Acids Res., 29:e45.

Pfaffl M.W., Tichopad A., Prgomet C., Neuvians T.P. (2004). Determination of stable housekeeping genes, differentially regulated target genes and sample integrity: BestKeeper-Excelbased tool using pair-wise correlations. Biotechnol. Lett., 26: 509-515.

Schmittgen T.D., Livak K.J. (2008). Analyzing real-time PCR data by the comparative C(T) method. Nat. Protoc., 3: 1101-1108.

Sirotkin A.V., Kisová G., Brenaut P., Ovcharenko D., Grossmann R., Mlync e k M. (2014). Involvement of microRNA Mir15a in control of human ovarian granulosa cell proliferation, apoptosis, steroidogenesis, and response to FSH. MicroRNA, 3: 29-36.

Ti a n F., L u o J., Z h ang H., Ch ang S., S ong J. (2012). MiRNA expression signatures induced by Marek's disease virus infection in chickens. Genomics, 99: 152-159. 
Vandesompele J., De Preter K., Pattyn F., Poppe B., Van Roy N., De Paepe A., S p e le m a n F. (2002). Accurate normalisation of real-time quantitative RT $\times$ PCR data by geometric averaging of multiple internal control genes. Genome Biol., 18:3, Research 0034.

Wang Q., Ga o Y., Ji X., Qi X., Q in L., Ga o H., Wang Y., Wang X. (2013). Differential expression of microRNAs in avian leukosis virus subgroup J-induced tumors. Vet. Microbiol., 162: $232-238$.

Wang W., Wu K., Jia M., S un S., Kang L., Zhang Q., Tang H. (2018). Dynamic changes in the global microRNAome and transcriptome identify key nodes associated with ovarian development in chickens. Front. Genet., 9: 491.

Wu N., Gaur U., Zhu Q., Chen B., Xu Z., Zhao X., Yang M., Li D. (2017). Expressed microRNA associated with high rate of egg production in chicken ovarian follicles. Anim. Genet., 48: 2005-2016.

Xu Q., Zhang Y., Chen Y., Tong Y.Y., Rong G.H., Huang Z.Y., Zha o R.X., Zha o W.M., W u X.S., Ch ang G.B., Ch e n G.H. (2014). Identification and differential expression of microRNAs in ovaries of laying and broody geese (Anser cygnoides) by Solexa sequencing. PLosOne, 9(2):e87920.

Received: 24 IV 2019

Accepted: 1 X 2019 